\title{
Unusual presentation of uterine rupture in modern obstetrics
}

\author{
Nandish Manoli, Surakshith L. Gowda*
}

Department of Obstetrics \& Gynecology, JSS Medical College and Hospital, Mysore, Karnataka, India

Received: 22 October 2014

Accepted: 1 November 2014

\author{
*Correspondence: \\ Dr. Surakshith L. Gowda, \\ E-mail: surakshithlgowda@gmail.com
}

Copyright: () the author(s), publisher and licensee Medip Academy. This is an open-access article distributed under the terms of the Creative Commons Attribution Non-Commercial License, which permits unrestricted non-commercial use, distribution, and reproduction in any medium, provided the original work is properly cited.

\begin{abstract}
Rupture of uterus in modern obstetrics is rare. If not diagnosed in time maternal morbidity and mortality are very high. We are reporting a case initially referred as gravida abortion $_{1}$ with breech presentation in active labour and was taken up for emergency LSCS, and on opening up a posterior uterine wall rupture was noted, later it was noted that the patient misled us by hiding her obstetric history of undergoing repeated MTPs. This case has been published in view of misleading history, variable clinical presentation \& to emphasize the need of definitive surgeries in order to save the life of the individual.
\end{abstract}

Keywords: Rupture of uterus, Misleading history, Repeated MTPs, Definitive surgeries

\section{INTRODUCTION}

It was Bandl who first described the condition uterine rupture in $1875 .{ }^{1}$ Uterine rupture is the most serious complication when not noticed in time for both mother and the baby. Despite known predisposing factors, uterine rupture is a rare event, and it is impossible to predict those women in whom it will actually occur. ${ }^{2}$ Meta-analysis of pooled data from 25 studies in the peerreviewed medical literature published from 1976-2012 indicated an overall incidence of pregnancy-related uterine rupture of 1 per 1416 pregnancies $(0.07 \%)$. When the studies were limited to a subset of 8 that provided data about the spontaneous rupture of unscarred uteri in developed countries, the rate was 1 per 8,434 pregnancies $(0.012 \%))^{3}$ In another 12 year study on intrapartum rupture of an unscarred uterus from January 1, 1983, through December 31, 1994, the incidence was 1 in 16849 deliveries. $^{4}$

\section{CASE REPORT}

Mrs. XY aged 27 years gravida 2 ; abortion $_{1}$ with no living issues married for 5yrs was referred to JSS medical college hospital, Mysore from a PHC in view of breech presentation with 38 weeks of gestation in active labour. On going in to the history it was noted that the patient was in labour for 18 hours with leak PV for 12 hours. On examination the patient looked anxious, dehydrated, mildly febrile with a pulse rate of $120 \mathrm{~b} / \mathrm{min}$ and a BP of 100/70 mmHg. The abdomen was transversely stretched $\&$ and the fetus in utero appeared to be in transverse lie \& was confirmed by PV \& bedside ultrasound examination.

An emergency LSCS was planned \& the per-op findings were that the lower uterine segment was thinned out \& stretched, liquor was absent. There was some difficulty in extraction of the baby so the incision was extended upwards on the right side, to our surprise a $4 \mathrm{~cm}$ vertical rent was noted in the posterior aspect of the uterine wall extending up to the vagina. Bladder injury was ruled out. The findings \& the further surgery was discussed with patient's attenders \& consent regarding the need for hysterectomy was obtained. In view of profuse bleeding from the rent \& irregular tear it was decided upon hysterectomy, later on examination of the specimen it was found to be a septate uterus. 2 pint of PRBC \& 1 pint of NS, RL, and Hemacel was transfused intraoperatively and 1 PRBC was transfused in the post-operative period. A term male baby was extracted by breech with birth 
weight of $2.9 \mathrm{~kg} \&$ APGAR of $6 \& 8$ at $1^{\text {st }}$ and $5^{\text {th }}$ minute respectively. The baby cried after tactile stimulation and was shifted to NICU for stage 1 birth asphyxia

Later the detail regarding the obstetric history was elicited. It was surprising that the patient had a bad social history \& was staying away from the husband and was actually gravida 5 ; abortion $_{4}$ and had undergone repeated MTPs at a local hospital. After 6 days the baby was handed over to the mother. The post-operative period was uneventful and the patient was discharged on the $10^{\text {th }}$ post-operative day.

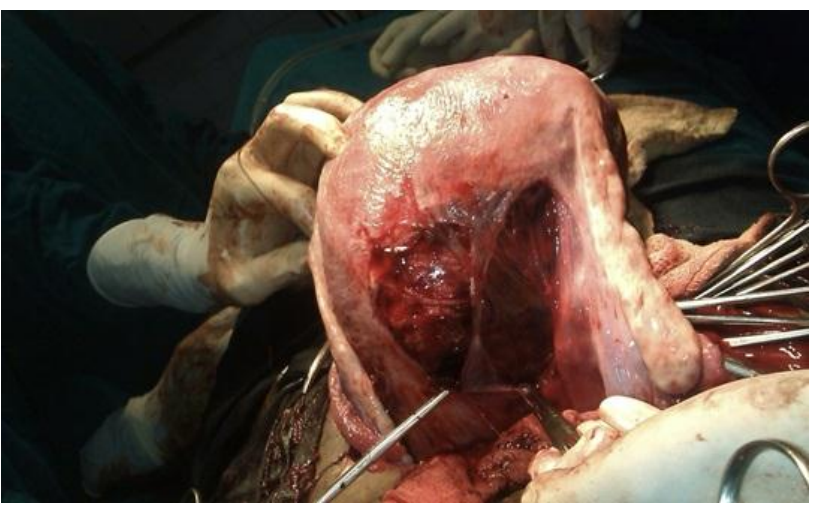

Figure 1: Showing vertical rent on the posterior uterine wall.

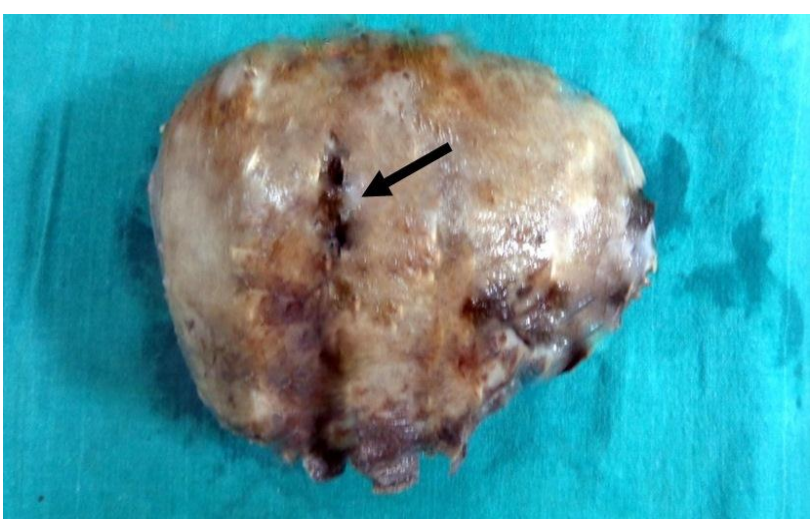

Figure 2: The arrow pointing towards the vertical rent on the posterior surface of the uterus.

\section{DISCUSSION}

Uterine rupture is a condition with breach in the uterine wall which may be complete or incomplete depending upon the intactness of the peritoneum ${ }^{5}$. Based upon the etiological factors uterine rupture could be divided into those of the intact/unscarred uterus and of the scarred uterus. Traumatic \& spontaneous rupture of the intact uterus characteristically involves the distended \& weakened lower uterine segment. This portion of the uterus is particularly vulnerable irrespective of whether the causative factor is traumatic, extrinsic force or weakness of the uterine wall resulting from numerous pregnancies. ${ }^{1}$
Causes of uterine rupture in unscarred/ intact uterus may be due to grand multiparity, injudicious use of oxytocin, uterine instrumentation and manipulation, congenital abnormalities of uterus, uterine distension due to polyhydramnios, multiple pregnancy and fetal macrosomia, neglected labour, manual removal of placenta. Breech extraction \& difficult forceps deliveries are the most frequent traumatic manipulations preceding uterine rupture, excessive fundal uterine pressure to aid delivery can also be a contributing factor in traumatic rupture. ${ }^{1,3,5,6}$

Spontaneous rupture of unscarred uterus is most frequently seen in advanced maternal age ${ }^{5} \&$ parity where the obstructed labour due to transverse lie, hydrocephalus, brow presentation, compound presentation, vertex presentation are neglected. ${ }^{1,5}$ In patients experiencing traumatic or spontaneous laceration of intact uterus exhibit rapidly progressive \& severe symptoms and most frequently present with shock \& vaginal bleeding so it should be diagnosed with high clinical suspicion to avoid adverse maternal and fetal outcome.

In a scarred uterus, the vast majority of uterine dehiscence and ruptures will occur via the uterine scar. The atrophic, inelastic nature of the scar renders it less adaptive to forces in labour, predisposing to scar rupture ${ }^{2}$. Trial of labour on scarred uterus and the use of uterotonics during labour are the most frequent cause of uterine rupture in the developed world ${ }^{5}$. Classical type of cesarean scar ruptures more readily than lower segment scars. From a study of uterine rupture following cesarean section that the risk of rupture of the lower segment scar is about $1 / 10^{\text {th }}$ that of classical scar. The type of previous uterine scars include lower segment, classical, myomectomy, hysterotomy, cornual resection \& emphasis on the need for careful observation should be given to the patients with previous scar when they are given TOL. ${ }^{1}$

Signs \& symptoms of uterine rupture are associated with sudden or atypical maternal abdominal pain, tachycardia, vaginal bleeding with shock, ${ }^{1,3}$ loss of uterine contractility $\&$ rarely blood stained urine ${ }^{6}$ may be the clinical presentation. Prolonged, late or recurrent variable decelerations or fetal bradycardias are often the first signs of uterine rupture. ${ }^{3}$ Posterior uterine rupture is extremely rare, conventional signs may be absent, and women may compensate well for massive concealed hemorrhage. ${ }^{2}$ As soon as the uterine rupture is diagnosed management must include resuscitation for the mother until surgical intervention and arrest of hemorrhage is done ${ }^{6} \&$ to deliver the fetus 10-37 minutes after uterine rupture necessarily to prevent serious fetal morbidity and mortality. 3,6

After the fetus has been delivered, the type of surgical treatment for the mother should depend on the following factors: type, extent of uterine rupture, degree of hemorrhage, general condition of the mother $\&$ her desire 
for further childbearing. Uterine bleeding is typically most profuse when the uterine tear is longitudinal rather than transverse. Conservative surgical management by uterine repair should be considered when there is: desire for future childbearing, no extension of the tear to the broad ligament, cervix, or paracolpos, easily controllable hemorrhage. Hysterectomy should be considered the treatment of choice when intractable uterine bleeding occurs or when the uterine rupture sites are multiple, longitudinal, or low lying ${ }^{3}$. Some of the complications of rupture uterus are wound infection, bladder injury, vesicovaginal fistula, ${ }^{5}$ urinary tract infection, deep vein thrombosis. ${ }^{7}$

According to WHO systematic review of maternal mortality and morbidity in the prevalence of uterine rupture in a systematic review of all available data since 1990-2005 Maternal mortality ranged between 1\% and $13 \%$, and perinatal mortality between $74 \%$ and $92 \%$. Reduction of the prevalence of rupture of unscarred uterus requires the following: reduction of unwanted pregnancies, particularly for women of high parity; accessibility of obstetric services including caesarean section for obstructed labour; where conventional caesarean section facilities are not accessible, innovative solutions such as symphysiotomy or caesarean section with local analgesia should be considered; and guidelines to ensure that misoprostol for labour induction is used in safe dosages. ${ }^{8}$

\section{CONCLUSION}

In this case the uterine rupture could be due to repeated MTPs with improper technique in instrumental evacuation with aggressive curettage which might have been a cause for a weak uterine wall along with a prolonged obstructed labour due to transverse lie. This case has been published in view of misleading history, variable clinical presentation, lack of proper medical care $\&$ the need of definitive surgeries in order to save the life of the individual.
Funding: No funding sources Conflict of interest: None declared

Ethical approval: Not required

\section{REFERENCES}

1. James D. Garnet. Uterine rupture in pregnancy: an analysis of 133 patients. Obstet Gynecol. 1964 Jun;23(6):898-905.

2. K. Navaratnam, P. Ulaganathan, M. A. Akhtar, S. D. Sharma, M. G. Davies. Posterior uterine rupture causing fetal expulsion into the abdominal cavity: a rare case of neonatal survival. Case Rep Obstet Gynecol. 2011;2011:426127.

3. Gerard G. Nahum, David Chelmow. Uterine rupture in pregnancy, 2012. Available at: http://reference.medscape.com/article/275854overview. Accessed 31 July 2012.

4. Miller DA, Goodwin TM, Gherman RB, Paul RH. Intrapartum rupture of the unscarred uterus. Obstet Gynecol. 1997 May;89(5 Pt 1):671-3.

5. Fofie CO, Baffoe P. Two-year review of uterine rupture in a regional hospital. Ghana Med J. 2010 Sep;44(3):98-102.

6. Qudsia Qazi, Zubaida Akhtar, Kamran Khan, Amer Hayat Khan. Woman Health; Uterus rupture, its complications and management in teaching hospital Bannu, Pakistan. Maedica (Buchar). 2012 Jan;7(1):49-53.

7. Esraa Hameed. Rupture uterus in pregnancy and labour in Al-Yarmouk teaching hospital. Iraqi $\mathbf{J}$ Community Med. 2010 Apr;23(2):110-3.

8. WHO. WHO systematic review of maternal mortality and morbidity: the prevalence of uterine rupture. BJOG. 2005 Sep;112:1221-8.

DOI: $10.5455 / 2320-1770 . i j r \operatorname{cog} 20141262$

Cite this article as: Manoli N, Gowda SL.

Unusual presentation of uterine rupture in modern obstetrics. Int J Reprod Contracept Obstet Gynecol 2014;3:1160-2. 